\title{
Gauge fixing in lattice QCD with multi-GPUs*
}

\author{
MARIO SCHRÖCK ${ }^{\dagger}$ \\ Institut für Physik, FB Theoretische Physik, Universität Graz, A-8010 Graz, \\ Austria \\ HANNeS Vogt \\ Institut für Theoretische Physik, Auf der Morgenstelle 14, 72076 Tübingen, \\ Germany
}

\begin{abstract}
Here we present the $c u L G T[$ code for gauge fixing in lattice gauge field theories with graphic processing units (GPUs). Implementations for SU(3) Coulomb, Landau and maximally Abelian gauge fixing are available and the overrelaxation, stochastic relaxation and simulated annealing algorithms are supported. Performance results for single and multi-GPUs are given.
\end{abstract}

PACS numbers: 11.15.Ha, 12.38.Gc

\section{Introduction}

Gauge fixing in lattice QCD is necessary in order to, e.g., compare lattice results to continuum physics in a given renormalization scheme at a given scale. The popular Landau gauge requires the four dimensional gradient of the gauge field to vanish at each space-time point of the lattice. The latter continuum condition translates to a large scale optimization problem in lattice gauge field theories. Finding its maxima is very expensive in terms of computational costs and a possible acceleration by high performance fine grained parallel architectures, like graphic processing units (GPUs), is highly desirable. Here we present a code written in CUDA which has been developed for the purpose of lattice gauge fixing on GPUs. The code makes strong use of template classes and algorithm abstraction to increase

\footnotetext{
* Presented at Excited QCD 2013, Bjelasnica Mountain, Sarajevo.

$\dagger$ Speaker

1 Www. cuLGT.com
} 
its flexibility and to extend its applicability to related problems in lattice gauge field theory.

Lattice QCD gauge fixing on GPUs was first presented in [1] and a detailed discussion of our code can be found in Ref. [2]. The authors of 3. use the Fourier accelerated deepest descent method for gauge fixing in lattice QCD.

In the following discussion we restrict ourselves to the example of Landau gauge fixing and we refer to 2 for the details of the other gauges and algorithms which are supported by $c u L G T$.

\section{Lattice Landau gauge}

The continuum Landau gauge condition,

$$
\partial_{\mu} A_{\mu}(x)=0,
$$

is fulfilled if and only if the lattice gauge functional

$$
F^{g}[U]=\frac{1}{N_{c} N_{d} V} \mathfrak{R e} \sum_{\mu, x} \operatorname{tr}\left[U_{\mu}^{g}(x)\right],
$$

resides in a stationary point with respect to gauge transformations $g(x) \in$ $\mathrm{SU}\left(N_{c}\right)$. Here we denoted a gauge transformation of the link variables as

$$
U_{\mu}^{g}(x) \equiv g(x) U_{\mu}(x) g(x+\hat{\mu})^{\dagger} .
$$

$N_{c}$ is the number of colors, $N_{c}=3$ for QCD, $N_{d}$ is the number of space-time dimensions, (here $N_{d}=4$ ) and $V$ is the total number of lattice points.

A measure $\theta$ of the Landau gauge precision is the average $L_{2}$-norm of the gauge fixing violation $\Delta(x)$, i.e., the discrete derivative of the continuum gauge fields

$$
\begin{aligned}
\Delta(x) & \equiv \sum_{\mu}\left(A_{\mu}(x)-A_{\mu}(x-\hat{\mu})\right)=0, \\
\theta & \equiv \frac{1}{N_{c} V} \sum_{x} \operatorname{tr}\left[\Delta(x) \Delta(x)^{\dagger}\right] .
\end{aligned}
$$

\section{The relaxation algorithms}

The idea of the relaxation algorithms is to sweep over the lattice site by site while optimizing the gauge functional locally. All sites of one of the two parity subsets (checker board decomposition) can be optimized at the same time because the newly generated local optimum depends on the nearest neighbors only. 
Instead of taking the complete global gauge functional into account,

$$
F^{g}[U]=\frac{1}{2 N_{c} N_{d} V} \mathfrak{R e} \sum_{x} f^{g}(x),
$$

the relaxation algorithm aims at optimizing the value of $F^{g}[U]$ locally, i.e., we search the maximum of

$$
f^{g}(x)=\mathfrak{R e} \operatorname{tr}[g(x) K(x)]
$$

for all $x$. Here we defined

$$
K(x):=\sum_{\mu}\left(U_{\mu}(x) g(x+\hat{\mu})^{\dagger}+U_{\mu}(x-\hat{\mu})^{\dagger} g(x-\hat{\mu})^{\dagger}\right) .
$$

For $\mathrm{SU}(2)$, the maximum thereof is given by

$$
g(x)=K(x)^{\dagger} / \sqrt{\operatorname{det} K(x)^{\dagger}}
$$

and for $\mathrm{SU}(3)$ one iteratively operates in the three $\mathrm{SU}(2)$ subgroups [4] and thereby optimizes the local $\mathrm{SU}(3)$ gauge functional.

\subsection{Overrelaxation}

Replacing the local gauge transformation $g(x)$ by $g^{\omega}(x), \omega \in[1,2)$ reduces the critical slowing down of the relaxation algorithm on large lattices [5]. In practice the exponentiation of the gauge transformation is done to first order.

\subsection{Stochastic relaxation}

The stochastic relaxation algorithm replaces the local gauge update $g(x)$ by $g^{2}(x)$ with probability $p$ and can speed up the convergence on large lattices.

\section{Single-GPU implementation}

We assign eight CUDA threads to each lattice site of a given parity in order to calculate and apply the local gauge update (9). The two parity sublattices are treated consecutively and the relaxation algorithm is iterated until the requested gauge precision $\theta$ has been reached. A variable data storage pattern for the gauge fields is adopted in order to meet the memory coalescing constraints of the hardware. In Fig. 1 we compare the performance of the code on different NVIDIA devies. 


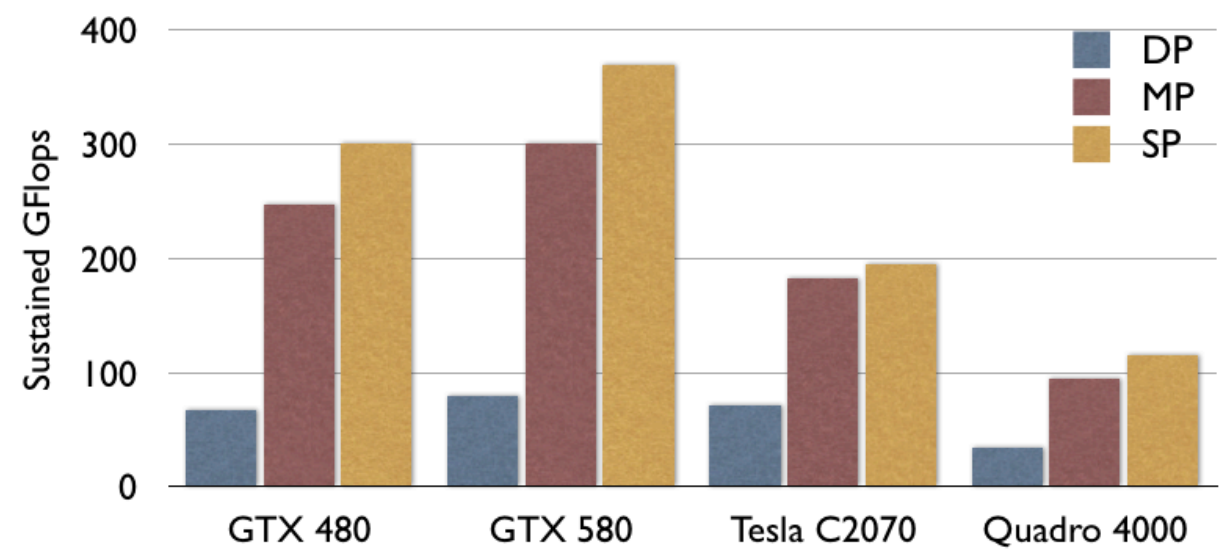

Fig. 1. Performance of the overrelaxation kernel on different NVIDIA devices in single (SP), mixed (MP) and double precision (DP) on a $32^{4}$ lattice.
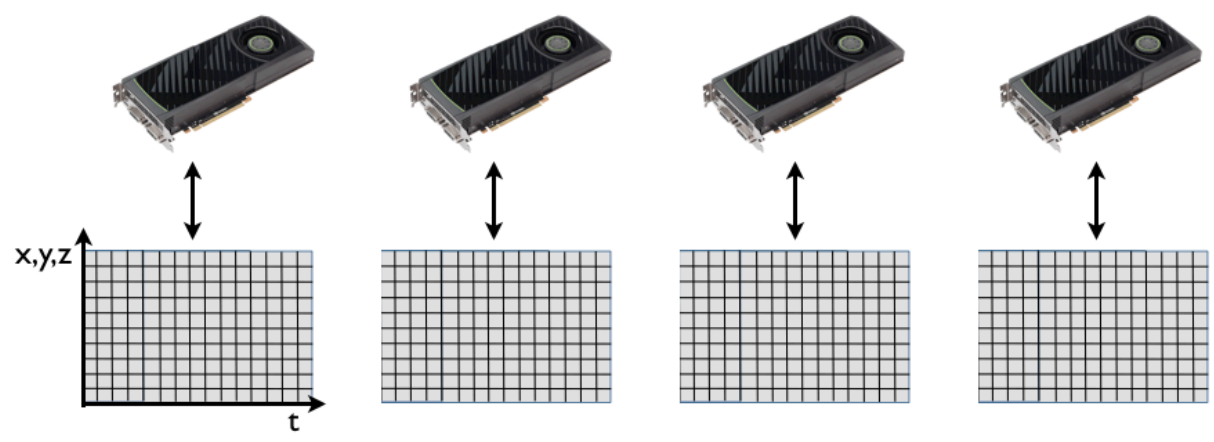

Fig. 2. The four dimensional lattice is split along the temporal axis and distributed to the devices. The bottle neck is the communication at the boundaries via the PCI-bus.

\section{Multi-GPU implementation}

For the multi-GPU implementation we decided for a decomposition of the lattice along the temporal axis, see Fig. 2. In each step of the iteration, the gauge links of the neighbor device in the temporal direction have to be exchanged via MPI in order to calculate the gauge update (9). After the gauge update has been calculated it has to be applied to all connected gauge links, therefore it has to be copied to the neighbor device. In detail, the following set of instructions has to be carried out on each device in order to transfer the links $U_{0}\left(t_{\max }\right)$ of device $i$ to device $i+1$ : 


\begin{tabular}{c|c|c|c|c|c}
$N_{s}^{3}$ & $\mathrm{D} 2 \mathrm{H}[\mu s]$ & $\mathrm{H} 2 \mathrm{D}[\mu s]$ & kernel $[\mu s]$ & $\mathrm{D} 2 \mathrm{H} /$ kernel & H2D / kernel \\
\hline 16 & 0.0398 & 0.0368 & 0.0209 & 1.90 & 1.76 \\
32 & 0.2543 & 0.2276 & 0.1443 & 1.76 & 1.58 \\
64 & 1.2510 & 1.1830 & 1.0489 & 1.19 & 1.13 \\
128 & 8.9597 & 8.7169 & 8.3041 & 1.08 & 1.05
\end{tabular}

Table 1. Time in microseconds needed to copy the data at the boundaries from device to host (D2H) and host to device (H2D) compared to the time needed to update one time-slice with the overrelaxation kernel. The two most right columns give the ratios.

1. cudaMemcpyDeviceToHost of $U_{0}\left(t_{\max }\right)$ (inactive parity)

2. MPI_Send of $U_{0}\left(t_{\max }\right)$ to device $i+1$ and MPI_Recv of $U_{0}\left(t_{\min }-1\right)$ from device $i-1$

3. cudaMemcpyHostToDevice of $U_{0}\left(t_{\min }-1\right)$

4. update $U_{\mu}\left(t_{\min }\right)$ (active parity) which affects $U_{0}\left(t_{\min }-1\right)$ (inactive)

5. cudaMemcpyDeviceToHost of $U_{0}\left(t_{\min }-1\right)$ (inactive parity)

6. MPI_Send of $U_{0}\left(t_{\min }-1\right)$ to device $i$ and $M P I_{-} R e c v$ of $U_{0}\left(t_{\max }\right)$ from device $i+1$

7. cudaMemcpyHostToDevice of $U_{0}\left(t_{\max }\right)$

In order to hide to slow data exchange over the low-bandwidth PCI-bus, we perform asynchronous memory transfers: we overlap the data exchange on the boundaries with calculations in the inner part of the domain. In Tab. 1 we compare the time needed to update one time-slice in the inner part of the domain with the time needed to copy the data at the boundaries to the host memory and from the host memory to the neighboring device.

Fig. 3 confirms the predictions of Tab. 1 that linear weak scaling is achieved with asynchronous memory transfers.

\section{Summary}

The local relaxation algorithms for lattice gauge fixing are well suited to be accelerated with highly parallel architectures like GPUs. With the aim of retaining maximum performance in a multi-GPU implementation it is crucial to overlap the data exchange between the devices by calculations in the inner part of the domain. This allows for linear weak scaling and a maximum performance of $\sim 3.5$ Teraflops adopting 16 Tesla C2070 GPUs. 

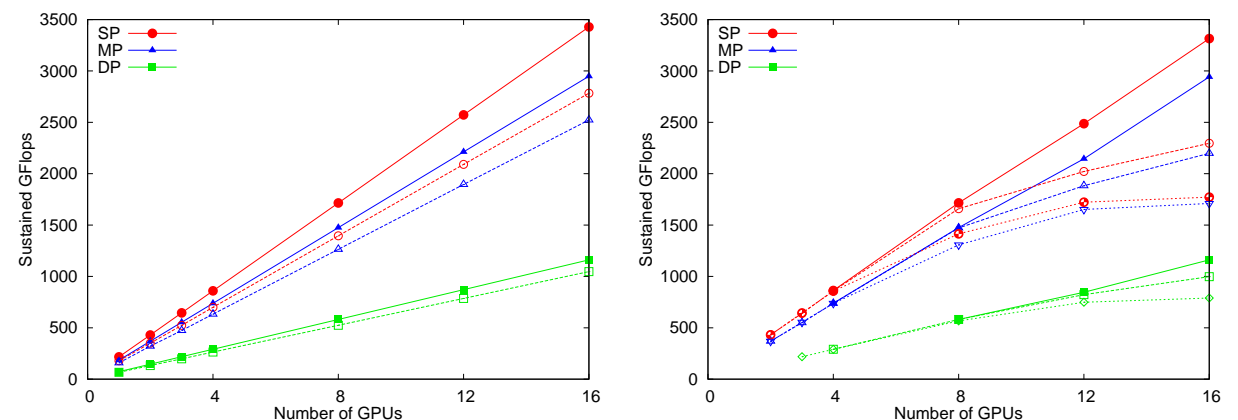

Fig. 3. Left: weak scaling on the NVIDIA Tesla C2070. The full symbols correspond to a lattice size of $64^{3} \times 32$ per GPU and the open symbols to $48^{4}$ per GPU. Right: strong scaling on the Tesla C2070. The spatial lattice volume is kept fixed at $64^{3}$ and the total temporal extent varies for the three lines (per precision) from the top downwards $N_{t}=256,128,96$.

\section{Acknowledgments}

Support by the Research Executive Agency (REA) of the European Union under Grant Agreement PITN-GA-2009-238353 (ITN STRONGnet) and by the Austrian Science Fund (FWF) through grant DK W1203-N16 is gratefully acknowledged.

\section{REFERENCES}

[1] M. Schröck, The chirally improved quark propagator and restoration of chiral symmetry, Phys.Lett. B711 (2012) 217-224. arXiv:1112.5107, doi:10.1016/ j.physletb.2012.04.008.

[2] M. Schröck, H. Vogt, Coulomb, Landau and Maximally Abelian Gauge Fixing in Lattice QCD with Multi-GPUs, Comp. Phys. Commun. 184 (2013) 19071919. arXiv:1212.5221, doi:http://dx.doi.org/10.1016/.

[3] N. Cardoso, P. J. Silva, P. Bicudo, O. Oliveira, Landau Gauge Fixing on GPUs, Comput.Phys.Commun. 184 (2013) 124-129. arXiv:1206.0675, doi:10.1016/ j.cpc.2012.09.007.

[4] N. Cabibbo, E. Marinari, A New Method for Updating SU(N) Matrices in Computer Simulations of Gauge Theories, Phys. Lett. B 119 (1982) 387. doi: 10.1016/0370-2693(82)90696-7.

[5] J. E. Mandula, M. Ogilvie, Efficient gauge fixing via overrelaxation, Phys.Lett. B248 (1990) 156-158. doi:10.1016/0370-2693(90)90031-Z. 